\title{
A Hybrid Control for Automatic Docking of Electric Vehicles for Recharging
}

\author{
Plamen Petrov, Clement Boussard, Samer Ammoun, and Fawzi Nashashibi
}

\begin{abstract}
In this paper, we present the architecture of an innovative docking station for electric vehicles recharging and a hybrid control scheme for automatic docking of the vehicles. This work is a part of on-going project concerning the development of a smart charging station for electric vehicles equipped with an automated arm, which connect the vehicle to the charging station, and an infrared beacon system for localizing the automatically maneuvering vehicle in the docking area. The proposed control scheme combines time-optimal (bang-bang) control with continuous time-invariant nonlinear control, which stabilizes the vehicle to a small neighborhood of the docking point. Simulation and experimental results illustrate the effectiveness of the proposed controller.
\end{abstract}

\section{INTRODUCTION}

$\mathrm{I}_{\mathrm{i}}^{\mathrm{N}}$ $\mathrm{N}$ recent years, the electric vehicles $(\mathrm{EV})$ has attracted increasing interest as the future means of transport motivated by the possibility of reducing fuel consumption and air pollution. The history of electric vehicles has known several market introduction attempts [1]. In the past, these attempts were condemned to failure for various reasons. The major obstacles to the adoption of EV by the drivers can be summarized in three points:

- Limited autonomy

- batteries recharging time

- The high cost due to the price of built-in batteries

A survey conducted during the 2010 Paris motor show in [2] shows that $89 \%$ of people are usually using their vehicles for less than $100 \mathrm{~km}$ per day, that $67 \%$ are ready to make a financial effort to acquire an electric car and that $41 \%$ prefers public transport downtown because it is difficult to find a parking place. The successful introduction of electric vehicles depends on several practical and commercial factors:

- The availability and the accessibility of recharging infrastructure.

The authors acknowledge the financial support from the AMARE project supported by the French agency for sustainable development ADEME.

P. Petrov is with the Faculty of Mechanical Engineering, Technical University of Sofia, 1000 Sofia, Bulgaria, (phone: 3592-965-3271; e-mail: ppetrov@tu-sofia.bg).

C. Boussard is with the Institut National de la Recherche en Informatique et Automatique (INRIA), 78153 Rocquencourt, France, (email: Clement.Boussard@inria.fr).

S. Ammoun is with Modulowatt ingénierie, 64 Bis avenue de New York, Paris, France (e-mail: Samer.ammoun@modulowatt.com).

F. Nashashibi is with the Institut National de la Recherche en Informatique et Automatique (INRIA), 78153 Rocquencourt, France, (email: Fawzi.Nashashibi@inria.fr).
- The ease and convenience of the connection procedure to the recharging stations since this maneuvers will be repeated several times per day.

- The adoption of urban policies that promote and reserves urban areas and parking places for EV.

- The emergence of new mobility services providers such as car-sharing or self-service vehicles. Indeed, new "electromobility operators" are starting to offer such services in urban areas like Autolib [3] in Paris and Car2go [4] in Germany and North America.

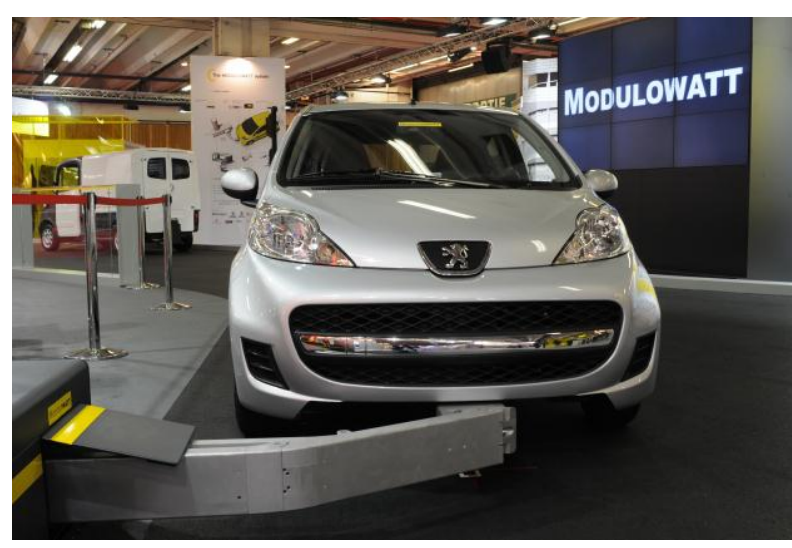

Fig. 1. Demonstration of the docking station for recharging during the Paris Motor Show 2010.

The AMARE project is developing a new concept that attempts to propose a systemic approach to the problem of the introduction of EVs in the city. Indeed, this new approach gives answers on multiple levels to meet the challenges associated with the introduction of electric vehicles in cities:

- Simplify and secure the maneuvers associated with the use of VE: automatic docking, recharging and payment.

- Reserve gradually urban surface parking to small VE using leverage from existing parking to finance the installation of new recharging stations.

In this paper we are going to concentrate our study on the EV automatic docking into the recharging station. The automatic docking constitutes a realistic application domain for research on automated vehicle since it is a low speed maneuver performed in a controlled environment. Thus, the AMARE approach consists on putting intelligence on the vehicle side, which is going to be automatically docked into 
the connector of the "dummy" recharging infrastructure.

During the last two decades, much research has been done in designing motion controllers for nonholonomic wheeled mobile robots and automated vehicles. The challenge of designing stabilizing controllers for nonholonomic mechanical systems is due to the limitation imposed by Brockett's necessary condition for point stabilization of such systems by means of smooth and even continuous timeinvariant feedback law. Traditional approaches such as linearization of the system equations about a point cannot be applied because the linearized system is not controllable. Despite the limitation imposed by Brockett's condition, two basic approaches for point stabilization have been proposed: a) discontinuous controllers [5] which are able to provide exponential stability; b) smooth time-varying controllers [6], where exponential stability of the closed-loop system is not possible, and sometimes this results in an unacceptable amount of time for stabilizing the system from a practical point of view. The problem of stabilizing nonholonmic systems to an equilibrium manifold by using time-invariant feedback has been also considered, [7]. To obtain faster convergence, hybrid controllers for stabilization to a small neighborhood of the origin have been proposed in $[8,9]$ where the problem of stabilizing is seen as an extension of the tracking problem. These controllers operate by switching between various control functions, depending on the fact whether the configuration coordinates are outside or inside a ball containing the origin. From a practical view point, this strategy may be quit acceptable, because of quick steering of the system to a small neighborhood of the origin. In [10], a bang-bang controller based on linear double integrator system is proposed to the problem of automated vehicle steering control, when the lateral vehicle dynamics is taken into account. An optimal control for route tracking with a bounded-curvature vehicle is proposed in [11], which is based on a kinematic vehicle model.

In this paper, we present the architecture of an innovative docking station for electric vehicles recharging (Fig. 1), and a hybrid control scheme for automatic docking. The control has a cascade structure and stabilizes the vehicle to a small neighborhood of the docking point (practical stabilization). This controller operates by switching between time-optimal (bang-bang) control and continuous nonlinear control law assuming that the front wheel steering dynamics is represented as a known stable second order linear system. The rest of the paper is organized as follows: In Section II, a short description of the system is given. The design of the proposed controller is given in Section III. Some simulation and experimental results are presented in section IV. Section $\mathrm{V}$ concludes the paper.

\section{DESCRIPTION OF THE SYSTEM}

The connection procedure for automatic charging is initiated by the driver through a Human-Machine Interface (HMI) by a touch screen [12]. First of all, the vehicle and the recharging infrastructure initiate a dialogue through their wireless communication interfaces. Using the embedded camera and and the POSIT algorithm, the vehicle is estimating its relative position to a InfraRed LEDs pattern on the recharging stations. Through a rigid transformation, the vehicle is calculating its coordinates in a reference centered on the connection point. The control system for the docking is working until the final position is reached. The automated arm makes the electric coupling between the vehicle and the charging station (Fig. 2).

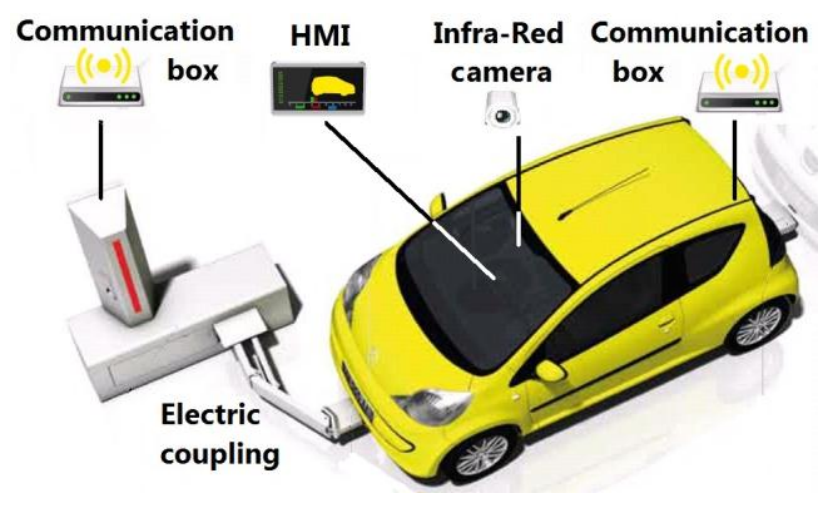

Fig. 2. System architecture

\section{VEHICLE CONTROL}

In this paper, we propose a cascade control scheme for the docking maneuver of a front wheel steered vehicle, which consists of two outer kinematics-based lateral and longitudinal control loops and two inner control loops for the steering angle and vehicle velocity, respectively. The designed kinematics-based control laws determine the desired values of the corresponding inner control loops. According the specifications of the project, the precision required for the docking maneuver is $\pm 10 \mathrm{~cm}$ in longitudinal and lateral direction around the docking point, and $\pm 15 \mathrm{deg}$ in orientation. In this case, we are interested in designing feedback control laws which achieves practical stabilization of the vehicle to a small neighborhood of the docking point

\section{A. Lateral time-optimal control}

A plan view of a front wheel steered vehicle during the docking maneuver is shown in Fig. 3.

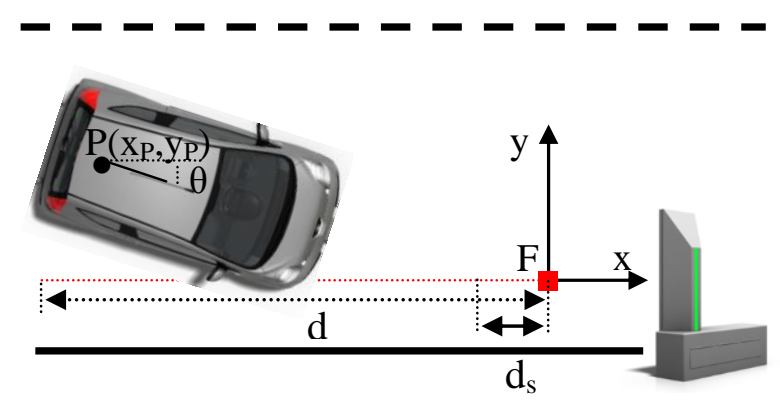

Fig. 3. Geometry of the docking maneuver. 
The vehicle is assumed to have planar motion. It is also assumed that the wheels are non-deformable and roll without slipping, which means that the velocity vectors of the wheel centers are in the orientation of the wheels. This assumption is reasonable for a slow moving vehicle (in the docking area, the speed is less than $1 \mathrm{~m} / \mathrm{s}$ ). The wheel base of the vehicle (the distance between the front and rear wheel axles) is denoted by $l$. To describe the position and orientation in the plane, an inertial frame $F x y$ is attached to the dock. The $F x$ axis is oriented in the direction of the desired motion of the vehicle to the recharging station. The coordinate of a reference point $P$, which is placed in the center of rear vehicle axle, with respect to $F x y$ are denoted by $\left(x_{P}, y_{P}\right)$. The angle $\theta$ is the orientation angle of the vehicle with respect to the frame Fxy. The steering angle of the front wheels is denoted by $\alpha$. The kinematic model of the vehicle in the plane can be described by the following nonlinear differential equations

$$
\begin{aligned}
& \dot{x}_{P}=v_{P} \cos \theta \\
& \dot{y}_{P}=v_{P} \sin \theta \\
& \dot{\theta}=\frac{v_{P}}{l} \tan \alpha
\end{aligned}
$$

where $v_{P}$ is the velocity of the reference point $P$.

We define a docking distance $d$, which for simplicity coincides with the $F x$ axis of the inertial frame $F x y$, as well and a vehicle stopping distance $d_{s}$, (Fig.3).

First, we present a time optimal (minimum-time) control for stabilization of the straight-line motion of the vehicle moving with constant velocity. The design of the control law is based on the nonlinear model given by the second and third equation of (1). The steering angle $\alpha$ of the front wheels is constrained by magnitude and takes the values

$$
\alpha= \pm \alpha_{c}
$$

where $\alpha_{c}$ is a positive constant. In particular, $\alpha_{c}$ can take the value of the maximum possible steering angle $\alpha_{\max }$ allowed from the mechanical construction. From a practical point of view, we are interested in restriction of $y_{P}$ and $\theta$ in the following intervals

$$
y_{P} \in\left[-\frac{\tan \alpha_{c}}{l}, \frac{\tan \alpha_{c}}{l}\right] ; \quad \theta \in\left[-\frac{\pi}{2}, \frac{\pi}{2}\right] .
$$

The time optimal control with bounded input is a bangbang control, which can take only two allowed extreme values. It can be expressed as a state feedback defined in terms of suitable switching curve in the phase plane.

Consider the subsystem composed of the second and third equation of (1). By eliminating the time $t$ between the two equations, after integration, the equation of the phase trajectories in the $y$ - $\theta$ phase plane is obtained as

$$
y_{P}=-2 \frac{l}{\tan \alpha_{c}} \sin \frac{\theta}{2}\left|\sin \frac{\theta}{2}\right|+C_{0}
$$

where $C_{0}$ is a constant equals to the distance along the $y_{P}$ axis between the coordinate center and the point where the phase trajectory intersects the $y_{P}$ axis. The constant $C_{0}$ in (4) can be determined by using the initial conditions $\left(y_{P O}, \theta_{0}\right)$ as follows

$$
C_{0}=y_{P 0}+\frac{2 l}{\tan \alpha_{c}} \sin \frac{\theta_{0}}{2}\left|\sin \frac{\theta_{0}}{2}\right| \text {. }
$$

The switching curve (for $C_{0}=0$ ) is described by the equation

$$
y_{P}=-2 \frac{l}{\tan \alpha_{c}} \sin \frac{\theta}{2}\left|\sin \frac{\theta}{2}\right| \text {. }
$$

Given the magnitude of the front wheel steering angle (2), the control objective is to move the state $\left(y_{P}, \theta\right)$ from initial point $\left(y_{P 0}, \theta_{0}\right)$, which belong to $(3)$, to the origin $\left(\left(y_{P}\left(t_{m}\right)\right.\right.$, $\left.\theta\left(t_{m}\right)\right)=(0,0)$ in the minimum time $t_{m}$. The time optimal (bang-bang) feedback control law can be stated as

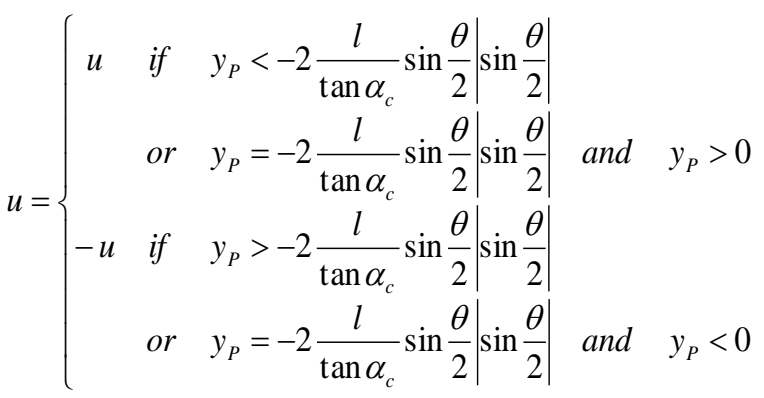

where $u=\frac{\tan \alpha_{c}}{l}$, and there is at most one control switching.

For initial states $\left(y_{P 0}, \theta_{0}\right)$ which are not on the switching curve (5), the control must switch between its extreme values so that any point in the phase plane which belongs to (3) can be moved to the origin in finite time. In this case, since the phase variable $\theta$ in the third equation of (1) depends linearly on time $t$, the time response $t_{m}$ of the system can be calculated as follows

$$
t_{m}=t_{1}+t_{2}=\left|\frac{\theta_{0}-2 \theta_{1}}{c}\right|
$$

where the time intervals $t_{1}$ and $t_{2}$ are obtained from the expressions

$$
t_{1}=\left|\frac{\theta_{1}-\theta_{0}}{c}\right| ; \quad t_{2}=\left|\frac{\theta_{1}}{c}\right|
$$

and represent the duration of motion of the point along the phase trajectories before and after the switching of the 
control, $c=\frac{v_{P}}{l} \tan \alpha_{c}$, and $\theta_{l}$ is determined from the relationships

$$
\theta_{1}=2 \arcsin \left[\sqrt{\left|\frac{\tan \alpha_{c}}{4 l} C_{0}\right|}\right] \operatorname{sgn}\left(u_{1}\right) .
$$

In practice, to apply the time optimal (bang-bang) control for path tracking, the dynamics of the inner control loop for the front wheel steering angle $\alpha$ must be taken into account, because the steering angle cannot be realized instantaneously. Since in this paper, we assume that the inner control loop for $\alpha$ has been already designed, for the purpose of the outer-loop control design, the front wheel steering dynamics is represented as a stable second order linear system. We approximate the dynamics for the steering angle by a pure time delay term, which can be expressed in the frequency domain as follows [13]

$$
e^{-\tau_{d}} \cong \frac{1}{1+\tau_{d} s+\tau_{d}^{2} s^{2} / 2}
$$

where $\tau_{d}$ is the time-delay. The idea behind this approach is to shift forward the switching curve developed for delayfree system in order to compensate for the dynamics of the inner control loop for $\alpha$. The new bang-bang control for the time-delay system which takes into account the steering dynamics is given as follows

$$
u=\left\{\begin{array}{lll}
u & \text { if } \quad y_{P}<-2 \frac{l}{\tan \alpha_{c}} \sin ^{2} \frac{\theta}{2}+4 \frac{l}{\tan \alpha_{c}} \sin ^{2}\left(\frac{\theta-\tau_{d}}{2}\right) \\
& \text { or } \quad y_{P}=-2 \frac{l}{\tan \alpha_{c}} \sin ^{2} \frac{\theta}{2}+4 \frac{l}{\tan \alpha_{c}} \sin ^{2}\left(\frac{\theta-\tau_{d}}{2}\right) \text { and } y_{P}>0 \\
-u & \text { if } \quad y_{P}>2 \frac{l}{\tan \alpha_{c}} \sin ^{2} \frac{\theta}{2}-4 \frac{l}{\tan \alpha_{c}} \sin ^{2}\left(\frac{\theta+\tau_{d}}{2}\right) \\
& \text { or } \quad y_{P}=2 \frac{l}{\tan \alpha_{c}} \sin ^{2} \frac{\theta}{2}-4 \frac{l}{\tan \alpha_{c}} \sin ^{2}\left(\frac{\theta+\tau_{d}}{2}\right) \text { and } y_{P}<0
\end{array}\right.
$$

In order to obtain better robustness in the presence of unknown steering dynamics and to avoid highly oscillatory behavior of the closed-loop system, it is desirable to switch to a continuous control in a neighborhood of the origin defined by

$$
\left|y_{p}\right| \leq y_{p L} \quad \text { and } \quad|\theta| \leq \theta_{L}
$$

where $y_{P L}$ and $\theta_{L}$ are positive constants. In this case, the control law has the form

$$
u=-\frac{\tan \left(k_{y} y_{p}+k_{\theta} \theta\right)}{l}
$$

where $k_{y}$ and $k_{\theta}$ are positive gains.

Finally, the time optimal control law (12) and the control
(14) can be blended so that, the quasi-optimal control operates by switching between the two control laws depending on the fact whether the state coordinates $\left(y_{P}, \theta\right)$ are outside or inside the neighborhood of the origin defined by (13). It should be noted that in the implementation, the control is also restricted with respect to $\alpha$ by the bound (2). The designed kinematics-based path tracking control law (outer control loop) determines the desired values of the inner control loop for $\alpha$.

\section{B. Longitudinal control}

The vehicle longitudinal control during the docking maneuver (when the distance between the vehicle and the docking station is less than the docking distance $d$ ), depends on the fact, whether $d>\left|x_{P}(t)\right|>d_{s}$ or $\left|x_{P}(t)\right| \leq d_{s}$. In the first case, the vehicle moves at a constant speed $v_{P}=$ cte. When $x_{P}(t)$ becomes equal or less than the stopping distance $d_{s}$, we assign a reference trajectory for $x_{p}$ in the form of a nonlinear differential equation

$$
\dot{x}_{r}+k_{x} x_{r}^{q}=0
$$

where $k_{x}$ and $q$ are positive constants. The constant $q$ belongs to the interval $1 / 2<q<1$. A specific feature of the proposed reference trajectory is that, $\dot{x}_{r}=0$ when $x_{r}=0$, $\left|\ddot{x}_{r}\right|$ is bounded, and $x_{r}$ becomes equal to zero in a finite time.

Using the first equation of (1) and (15), an inverse dynamics type feedback control is proposed in the form

$$
v_{P}=-\frac{1}{\cos \theta} k_{x} x^{q}
$$

The designed kinematics-based control law (16) determines the desired values for the inner control loop for the vehicle velocity $v_{P}$.

\section{SIMULATION AND EXPERIMENTAL RESUltS}

To illustrate the effectiveness of the proposed controller, several simulations are carried out in order to evaluate the vehicle behavior and docking accuracy. In the simulation using MATLAB, the longitudinal vehicle base was chosen to be $1.5 \mathrm{~m}$. The docking distance and stopping distance were chosen to be $6 \mathrm{~m}$ and $0.75 \mathrm{~m}$, respectively. The initial position and orientation of the vehicle were $x_{P}(0)=0 m, y_{P}(0)=$ $0.825 \mathrm{~m}$ and $\theta(0)=0.1 \mathrm{rad}$. The initial vehicle speed were $v_{P}$ $=0.5 \mathrm{~m} / \mathrm{s}$. The steering angle were constrained by magnitude $\alpha_{c}=\pi / 9 \mathrm{rad}$. The gains of the controller were chosen to be $k_{x}$ $=0.7, k_{y}=1$, and $k_{\theta}=2.25$. In accordance with the dynamics of the inner control loop for $\alpha$, the time-delay were determined to be $\tau_{d}=0.075$. The gain $b$ in the reference equation (15) were chosen to be $q=0.85$.

In the first simulation, (Fig. 4) we can see the phase plane trajectory of the system according to the control law (12). 


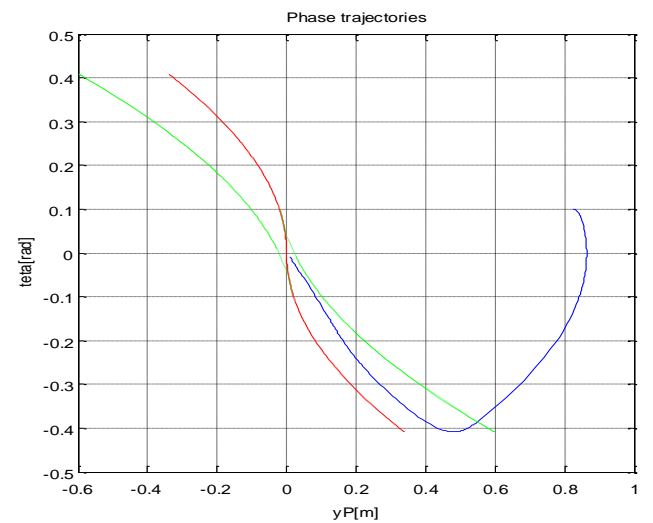

Fig. 4. Phase plane trajectories: system trajectory (blue solid line), switching curve for the delay-free system (red dashed line), switching curve for the system with time-delay (green dashed line).

The planar path, drown be the reference point $\mathrm{P}$ in the plane is shown in Fig. 5.

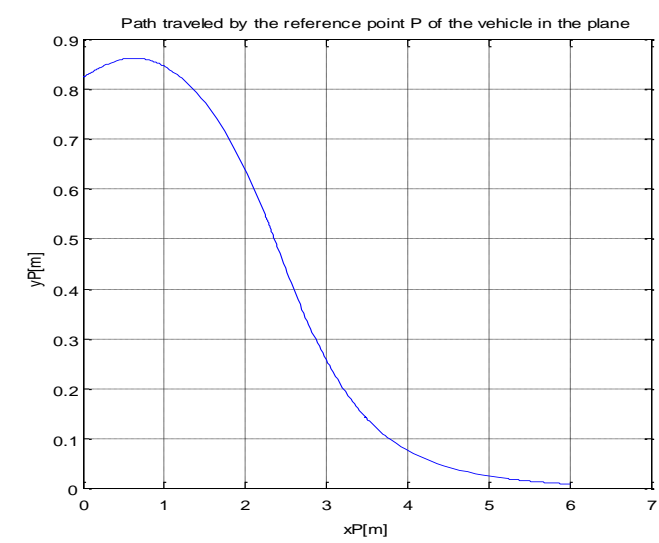

Fig. 5. A planar path drown by the vehicle reference point $P$.

The evolution in time of the longitudinal coordinate $x_{P}(t)$ of point $P$ during the docking maneuver is shown in Fig. 6 . The final value is equal of the assigned docking distance $d$.

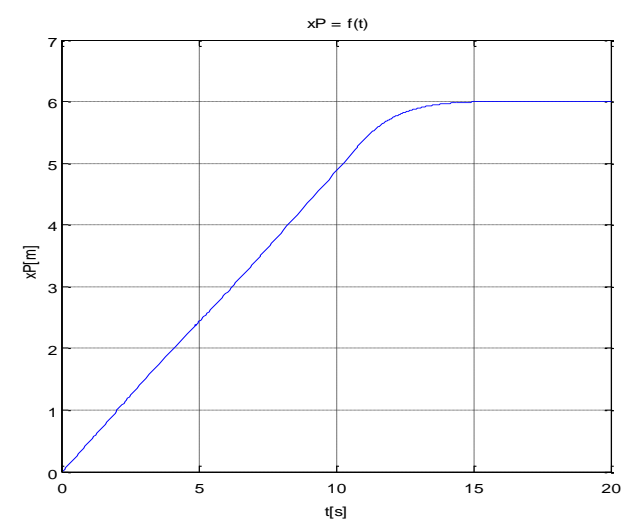

Fig. 6. Evolution in time of the longitudinal coordinate $x_{P}(t)$ of point $\mathrm{P}$.

From Fig. 7, we can see the evolution in time of the longitudinal coordinate $y_{P}(t)$ of point $P$ and the orientation angle $\theta(t)$ during the docking maneuver. There are steady- state errors which are in the conformity with the Brockett's obstruction for point stabilization of a nonholonomic system with smooth time-invariant feedback laws like (14) and (16).

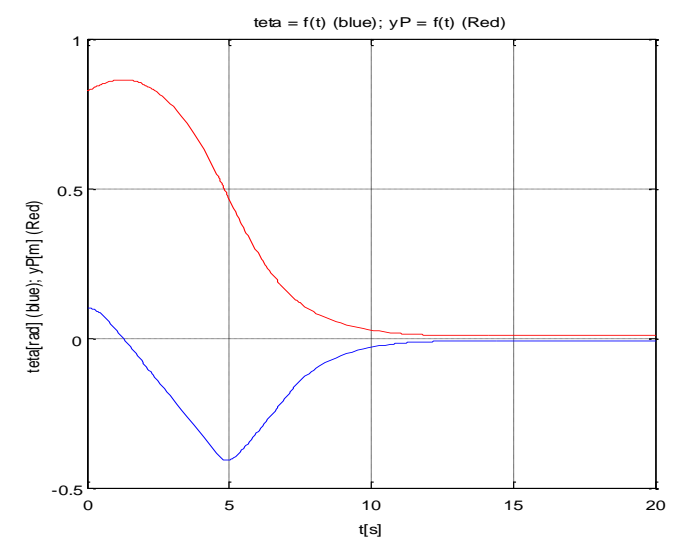

Fig. 7. Evolution in time of the longitudinal coordinate $y_{P}(t)$ of point $P$ and the orientation angle $\theta(t)$.

The evolution in time of the vehicle speed $v_{P}$ is shown in Fig. 8. When $x_{P}(t)<d_{s}$, the speed begins to decrease and converges to zero.

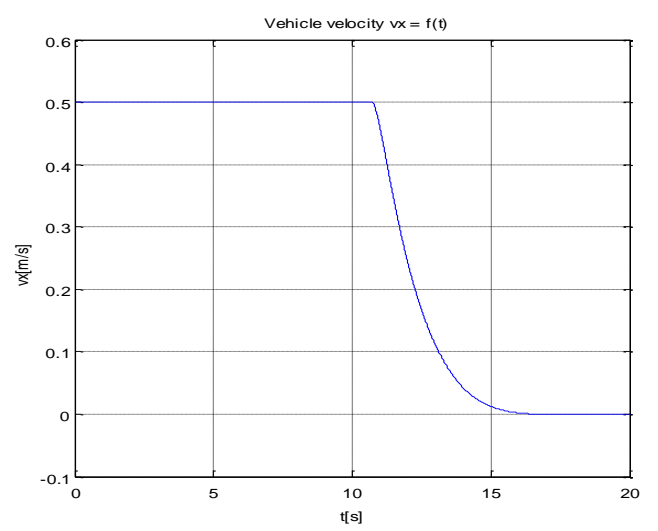

Fig. 8. Evolution in time of the vehicle speed $v_{P}$.

Evolution in time of the vehicle steering angle is shown in Fig.9.

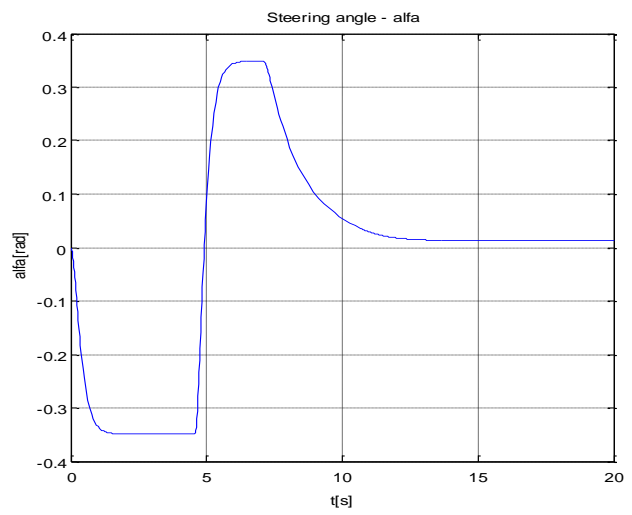

Fig. 9. Evolution in time of the vehicle steering angle $\alpha$. 
In the beginning, due to the time-optimal control, the angle $\alpha$ takes the maximum allowed values until the control scheme switches from bang-bang to smooth control.

Experiments with an automatic vehicle Peugeot 107 have been performed, (Fig. 10), where the position and orientation of the vehicle with respect to the docking station is obtained by means of a camera mounted on the vehicle and localization system developed by VALEO.

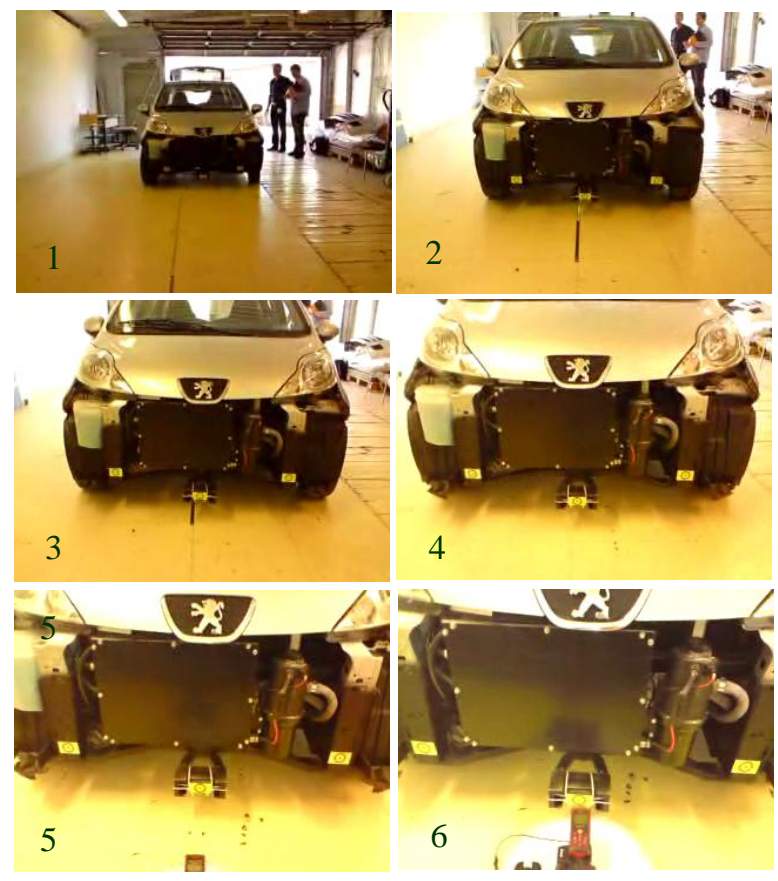

Fig. 10. Experiments in automatic vehicle docking.

Some preliminary results were also shown in the Paris Motor Show 2010 (Fig. 1). The results of the simulation and the experiments verify the validity of the proposed control scheme.

\section{CONCLUSION}

In this paper, an architecture of an innovative docking station for electric vehicles recharging and a hybrid control scheme for automatic docking of the vehicles have been presented. This work is a part of on-going project concerning the development of a smart charging station for electric vehicles. A cascade control scheme, which consists of two outer kinematics-based lateral and longitudinal control loops and two inner control loops for the steering angle and vehicle velocity, respectively has been proposed. The designed kinematics-based control laws determine the desired values of the corresponding inner control loops. Due to the limited dock area, the proposed control scheme combines timeoptimal (bang-bang) control with continuous time-invariant nonlinear control, which stabilizes the vehicle to a small neighborhood of the docking point, and in this way the vehicle performs docking maneuvers with minimum radius of turn. The dynamics of the inner control loop for the front wheel steering angle is taken into account and approximated by a pure time delay term, which is set experimentally. The continuous feedback control law is proposed in order to avoid chattering, when the lateral and orientation errors are less than some prescribed in advance values. Simulation results and experimental tests confirm the validity of the proposed control scheme. It should be highlighted that at present stage, this paper must be regarded as preliminary report of our work. Some issues remain open and deserve further investigation. Our future work will address the control problems associated with the simultaneous recharging of several mechanically linked vehicles in a tractor multi-trailer system (Fig. 11).

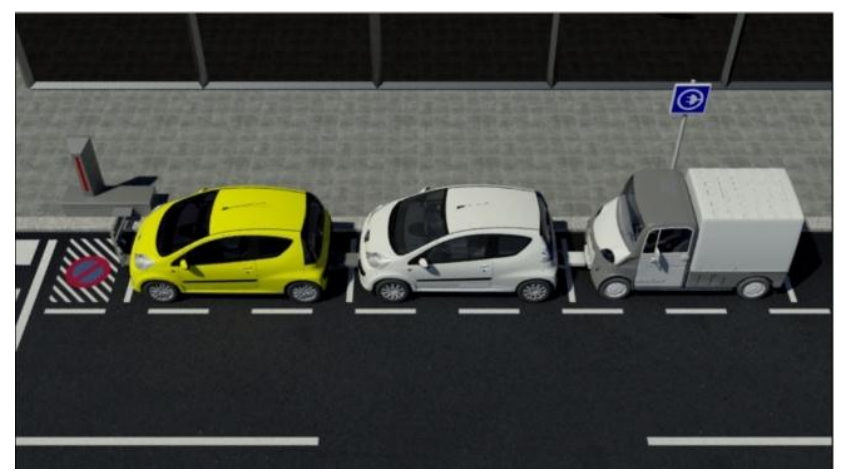

Fig. 11. Simultaneous recharging of several mechanically linked vehicles

\section{REFERENCES}

[1] C. Chan, "The state of the art of electric and hybrid vehicles", in 2002 Proc. IEEE, vol. 90, pp. 247-275.

[2] Poll done by the company Modulowatt for the newspaper '20 minutes' during the Paris Motor show 2011 http://modulowatt.com/pdf/MODULOWATT sondageVE Mondial2010.pdf

[3] Available at: http://www.autolib.fr.

[4] Available at: http://www.car2go.com

[5] N. Marchand and M. Almir, "Discontinuous exponential stabilization of chained form sytems", in 2002 Proc. IEEE CDC, pp. 350-355.

[6] C. Samson, "Control of chained systems: Application to path following and time time-varying point-stabilization of mobile robots", IEEE Trans. Aut. Control, vol.40, pp. 64-77, 1995.

[7] A. Bloch, M. Reyhanoglu, and N. H. McClarmoch, "Control and stabilization of nonholonomic dynamic systems", IEEE Trans. Aut. Control, vol.37, pp.1746-1757, 1992.

[8] P. Petrov, J. de Lafontaine, and M. Tetreault, " Hybrid feedback control for the parking problem of a load-haul-dump mine vehicle", in 1998 Proc. IEEE/RSJ IROS, pp. 1907-1912.

[9] R. Fierro and F. L. Lewis, " Control of a nonholonomic mobile robot: Backstepping kinematics into dynamics”, J. Rob. Systems, vol. 14, pp. 149-166.

[10] C. George and R. O'Brien, "Vehicle lateral control using double integrator control strategy", in 2004 Proc. Southeastern Symp. Syst. Theory, pp. 398-400

[11] P. Souares, A. Ballucuchi, and A. Biccchi, "Optimal feedback control for route tracking with a bounded-curvature vehicle", in Proc. 2000 IEEE ICRA, pp. 2473-2478.

[12] S. Ammoun, C. Boussard and L. Bouraoui, "Modulowatt: description d'un système innovant de recharge de véhicule électrique", in 6th National Conference on Control Architecture of Robots, 2011.

[13] B. Kuo, Automatic control systems, $5^{\text {th }}$ edition, Prentice-Hall, 1987. 